\title{
LA DOCTRINA RUSSELLIANA DEL PENSAMIENTO
}

\author{
Alejandro Tomasini Bassols
}

INSTITUTO DE INVESTIGACIONES FILOS6FIGAS

Universtoad Nacional Autónoma de Mexico

Es tan redundante o innecesario afirmar como absurdo negar la importancia del concepto de pensamiento, que no deja de ser un tanto sorprendente el que se hayan ofrecido a lo largo de la historia de la filosofía tan variadas concepciones de lo que éste es. En parte, tal vez, ello se debe a una cierta ambigüedad de la palabra 'pensamiento'. ${ }^{1}$ Dicha ambigüedad se manifiesta en dos aplicaciones legítimas del concepto que nos ocupa y que podríamos llamar respectivamente 'la aplicación colectiva' y 'la aplicación individual'. Es muy importante fijarse en que la dicotomía "colectivo-individual" no es equivalente a la dicotomía "público-privado". Aquí yo parto del principio de que no hay aplicaciones privadas de conceptos, si bien claramente reconozco la posibilidad y la actualidad de las aplicaciones individuales.

Probablemente la distinción "colectivo-individual" nos resultará más inteligible o inclusive familiar si la ponemos en conexión con filósofos concretos. Así, si por aplicación "colectiva" nos referimos a aquello que hablantes y escuchas comparten cuando se comunican entre sí, a lo que tienen en común oraciones de diversos idiomas, aquello que es aprehendido cuando se comprende una oración significativa del sistema de signos que se use, etc., el filósofo que naturalmente se nos viene a las mientes es Frege. Para Frege, en efecto, la investigación lógica por excelencia es la descripción detallada de la naturaleza, la estructura, las propiedades, etc., del pensamiento, entendido como el sentido de una oración, el cual forma parte de un reino objetivo del ser, a saber, el poblado por entidades como números, objetos lógicos, etc. Este reino del ser es obviamente diferente de los mundos físico y mental. Tenemos, pues, un primer significado de 'pensamiento'. Por otra parte, empero,

1 No estoy sugiriendo, desde luego, que una vez que dicha ambigüedad haya sido eliminada los problemas filosóficos que nos plantea la noción de pensamiento habrán quedado eo ipso resueltos. La previa labor aclaratoria no es sino la preparación del terreno para las genuinas especulación y discusión filosóficas, pero ella misma no es una investigación filosófica. 
'pensamiento' nos remite no a una entidad, abstracta o no, sino más bien a un proceso, a una actividad peculiar, esto es, a la actividad de la mente. En este segundo sentido, el filósofo por nombrar es, evidentemente, Descartes. Este último llega a hacer del pensar la esencia de la persona: yo soy una cosa que piensa y existo mientras y sólo mientras pienso (usando 'pienso' con liberalidad). Ahora bien, pensamiento fregeano y pensamiento cartesiano parecen agotar el concepto general de pensamiento. Lo que me propongo hacer en este trabajo es explorar algunos escritos de Russell, viz., los que van de los años 1900 a 1913, a fin de determinar si durante este período Russell logró elaborar una teoría completa del pensamiento y, en caso de que así fuera, de examinar dicha teoría críticamente.

Adelantándonos a la exposición, podemos decir desde ahora que la teoría russelliana del pensamiento tiene dos componentes fundamentales:

a) la teoría de la proposición;

b) la teoría del conocimiento directo ("acquaintance").

La teoría de la proposición — siendo 'proposición' el término russelliano equivalente al 'Gedanke' de Frege (si bien sus nociones no coinciden totalmente) - está expuesta, básicamente, en Los Principios de las Matemáticas, publicado en 1903. A pesar de haberla repudiado abiertamente desde 1907, Russell de hecho siguió manteniéndola en una versión ad hoc con su nueva ontología factual. Por otra parte, la teoría del "conocimiento directo" está virtualmente contenida en el esbozo de libro, nunca publicado por Russell, titulado Teoria del Conocimiento (1913), si bien está ya implícita en la Teoría de las Descripciones. Voy a dejar de lado las peripecias históricas del texto y me concentraré en su contenido una vez que haya presentado y discutido la primera parte de la doctrina de Russell. Hay que hacer antes, sin embargo, varias aclaraciones.

Es perfectamente legítimo preguntarse si hay o no un orden lógico o interno en la investigación acerca del pensamiento. Es evidente, por una parte, que la teoría del conocimiento es más amplia que la teoría de la proposición: conocemos proposiciones, pero también podemos hablar de conocimiento en relación con imágenes, sentimientos, etc. ¿Por qué entonces, de hecho, le dio Russell prioridad a la proposición sobre todos los objetos de conocimiento y por qué no desarrolló la teoría general del conocimiento sino diez años después de haber expuesto la doctrina de las proposiciones? Personalmente, soy de la opinión de que no hay un orden pre-establecido y que en el caso de Russell fueron consideraciones, por así llamarlas, 'pragmáticas' (o programáticas), las que lo lleva- 
ron a formular primero la doctrina del pensamiento como proposición. No sólo estaba ya a la mano parte de la gran obra de Frege y no sólo se dejaba sentir poderosamente, como veremos en un momento, la actividad filosófica de G. E. Moore en la misma dirección, sino que urgía ya poner una plataforma real para llevar a cabo el programa de fundamentación de nuestro conocimiento. El plan original de Russell, como es bien sabido, era justificar primero el conocimiento matemático reduciendo las matemáticas a la lógica, pasar después a los principios abstractos de la física y gradualmente al resto de las ciencias, mostrando de hecho que el conocimiento humano forma un todo coherente y continuo y que quizá también incluye a la ética y a la religión. Pero, insisto, lo que importaba era la justificación lógica del conocimiento. La explicación o la racionalización epistemológica, por lo tanto, podía esperar. Tenemos, pues, una razón, que podemos calificar de 'externa' a la temática, por la cual la teoría de la proposición recibió prioridad. Pero hay quizá otra razón, "interna" esta vez, que si bien es difícil atribuírsela a Russell mismo, sí justifica ex post facto su orden de investigación. La razón es que si bien podemos hacer un uso laxo de la noción de pensamiento de tal manera que incluyamos en él imágenes, recuerdos, sentimientos, sensaciones, emociones, etc., también es cierto que tenemos conciencia de dichos "objetos" y "eventos" mentales y que adquieren éstos el status de pensamiento en la medida en que pueden ser expresados, esto es, cn la medida en que podemos atraparlos lingüísticamente. Ahora bien, 'atrapar lingüísticamente' quiere precisamente decir no nombrar, sino "proposicionalizar" o, en el modo formal, "oracionalizar". En otras palabras, la clase fundamental de pensamiento es la de pensamiento proposicional. La proposición es el instrumento o el vehículo del conocimiento, es decir, no podemos ni siquiera afirmar que sabemos que algo nos acontece si no podemos en principio construir o enunciar proposiciones al respecto. Deseo, de paso, sugerir que, dado que la noción de conocimiento involucra a la de verdad, la conexión entre ambas queda más claramente establecida precisamente a través de la noción de proposición que, e.g., de la de creencia. Es decir, conviene, por razones en las que no entraré aquí, hacer de la proposición el portador primitivo y original de la verdad y la falsedad. Es sobre la base de la esencial bipolaridad de la proposición que el conocimiento empírico, tanto en el sentido fuerte de tener conciencia de algo externo a uno como en el sentido de poseer creencias verdaderas acerca de ello, justificadas, etc., es posible. Esto es en parte, creo, lo que se nos quiso decir en el Tractatus cuando Wittgenstein afirma que "Una figura lógica de los hechos es el pensamiento". ${ }^{2}$ Podemos, pues, decir, que cualquier cosa que aspire a

2 L. Wittgenstein. Tractatus Logico-Philosophicus, 3. 
pasar por conocimiento (y por ende por pensamiento) tiene que pasar a través del prisma constituido por la proposición. Habría, pues, una segunda razón para explicar por qué Russell inició su investigación en torno al pensamiento con una teoría de la proposición. Desgraciadamente, como dije, este argumento es un tanto anacrónico y es, además, poco russelliano, puesto que Russell siempre admitió como perfectamente posible el que objetos individuales, considerados aisladamente, fueran objetos de pensamiento $y$, asimismo, que pudiéramos nombrarlos sin necesidad de decir nada al respecto. Sobre estos problemas regresaré posteriormente, pero antes de iniciar la presentación de la teoría de Russell creo que será útil hacer una muy breve excursión histórica.

Mencioné a Frege para indicar un tipo especial de investigación en torno al pensamiento entendido como una "estructura lógica" gracias a la cual nuestras oraciones tienen vida. El artículo de Frege en el que dicha teoría es expuesta, titulado "The Thought: A Logical Inquiry", apareció en 1918. No obstante sus incuestionables méritos, si hemos de ser justos habremos de reconocer que, aunque de manera menos sofisticada y sistemática, otro filósofo había elaborado ya una concepción sorprendentemente semejante veinte años antes. Me refiero a G. E. Moore y a su artículo "The Nature of Judgment" (Mind 1898). Haré en lo que sigue una rápida presentación de la posición de Moore, porque ello nos servirá para comprender tanto lo que Russell dice como la problemática misma.

Paradójicamente, al igual que muchos filósofos británicos, Moore inicia su artículo con un ataque en contra del empirismo. Varias son las ideas que él quiere rebatir, pero dos destacan por su importancia. Está primero la idea de que pensamos mediante o en ideas, siendo éstas entidades subjetivas, y, segundo, está la tesis de que el significado de una idea es un concepto. Moore reconoce que podemos decir que tenemos ideas e inclusive que podríamos tal vez afirmar que cada quien tiene sus propias ideas, pero hace ver que ya no resulta inteligible sostener que cada quien tiene sus conceptos. Parecería que los conceptos son los mismos para todos y que son independientes de que los pensemos o no. Son objetivos y los aprehendemos cabal y completamente o no los captamos en lo absoluto. "Los conceptos son los objetos posibles del pensamiento (...). Es indiferente a su naturaleza el que alguien los piense o no. Ellos no son susceptibles de cambiar." ${ }^{3}$ Los conceptos son lo que constituye el contenido de nuestro pensamiento, entendido este último como proceso cognitivo, como pensamiento cartesiano. Pensar es entrar en contacto con conceptos y establecer relaciones entre ellos. Un juicio es

s G. E. Moore. "The Nature of Judgement", Mind 1898, p. 179. 
una combinación especial de conceptos. Es muy importante percatarse de que, en la perspectiva de Moore, las ideas, sean éstas lo que sean, son irrelevantes al o para el pensamiento. Los conceptos no son el resultado de abstracciones. Los conceptos son eternos y todos los juicios posibles están ya formados. Los juicios, a su vez, se dividen entre los existentes y los no existentes. Esto, obviamente, hace de la verdad algo aprehensible solamente de manera intuitiva. La consecuencia relevante para nuestro tema, sin embargo, es que dado que nosotros pensamos conceptos, los cuales constituyen juicios siendo estos últimos lo existente, es claro entonces que lo que nosotros pensamos es el mundo o la realidad. No hay intermediario alguno entre el sujeto o la mente y el mundo. Este último se compone de conceptos: para que, mental o verbalmente, digamos que el mundo es de tal o cual manera, necesitamos primero transformar a las cosas en conceptos. Pero los conceptos, a diferencia de las ideas, no son subjetivos ni mentales. Para Moore, repito, lo que nosotros pensamos es la realidad. Por ejemplo, la percepción, nos dice Moore, es la relación cognitiva que se mantiene con conceptos a través de los sentidos. La existencia no es sino una combinación especial de conceptos.

La teoría russelliana de la proposición es en cierto sentido una prolongación y un avance frente a la de Moore. Russell la elaboró en 1900, si bien ésta no fue publicada sino hasta 1903 . Un defecto que cabe de inmediato señalar en la teoría de Russell es que ésta no está sistemáticamente presentada (si bien emerge de consideraciones sobre sus componentes). Aparentemente siguiendo una distinción gramatical, Russell distingue entre nombres propios, adjetivos y verbos. De acuerdo con él, esas son las clases de expresiones filosóficamente importantes. Las demás expresiones son sincategoremáticas. Ahora bien, tanto nombres propios como adjetivos y verbos "significan" algo, a saber, "términos", pero los indican de manera distinta. No sólo eso: los términos mismos se clasifican en función de las partes de la oración que los denoten o refieran o signifiquen. Así, los nombres propios, es decir, todos aquellos sujetos gramaticales que no proceden lógicamente de adjetivos (como, por ejemplo, 'humanidad' de 'humano', 'inteligencia' de 'inteligente', etc.), indican cosas. Los adjetivos, por su parte, significan predicados, en tanto que los verbos significan relaciones. Predicados y relaciones son conceptos. Una característica curiosa e importante de los conceptos es que pueden aparecer en proposiciones no sólo qua conceptos, sino también como sujetos, fenómeno que nunca sucede con las cosas. Las cualidades y las relaciones también pueden ser nombradas, pero ese movimiento gramatical no puede, pace Frege, alterar su status lógico.` $\mathrm{El}$ hecho de

\footnotetext{
- Véase The Principles of Mathematics, Appendix A, $\S 483$.
} 
que hablemos de relaciones y propiedades no las transforma en objetos. Después de todo, los términos son lo que son independientemente de cómo hablemos de ellos. Para nuestros propósitos, el punto importante es el siguiente: cosas y conceptos se unen o fusionan para formar proposiciones. Antes de seguir adelante, será útil, sin embargo, hacer un par de aclaraciones con respecto a la noción de significado. En Los Principios de las Matemáticas, Russell distingue dos significados de 'significado':

1) un sentido sicológico;

2) un sentido lógico.

El significado sicológico de una expresión son las imágenes, asociaciones, líneas de conducta, reacciones, etc., que la palabra produce o inspira en quien la comprende. Es evidente que el significado en este sentido es totalmente irrelevante para las necesidades de la gramática filosófica. En éste, como en otros puntos, Russell todavía sigue a Moore. En el sentido lógico, el significado de unas palabras es sencillamente "su" término. Todos los términos se dividen en dos grupos, a saber, los que tienen ser y los que, además de ser, tienen existencia. El término "sirena" sólo tiene ser, en tanto que el término "Palacio Nacional" tiene ser y existencia. La pregunta ahora es: ¿qué conclusiones acerca de las proposiciones podemos extraer sobre la base de lo dicho hasta aquí?

Se desprende, en primer lugar, que las proposiciones no se componen de palabras, es decir, no son entidades lingüísticas. Las proposiciones se componen de términos. Constituyen, pues, una clase de entidades objetivas y complejas, ya que la proposición mínima ha de contener por lo menos dos términos, una cosa y un concepto. Tampoco son las proposiciones, por lo tanto, entidades mentales, aunque algunas de ellas podrían serlo. Por otra parte, paralelamente a la doble teoría del significado de las expresiones de nuestro lenguaje, Russell desarrolla una doble teoría de la aseveración. Por lo tanto, 'aseveración' tiene también dos sentidos:

a) un sentido sicológico, $\mathrm{y}$

b) un sentido lógico.

En el primer sentido, una proposición aseverada es simplemente la situación o el estado de cosas que el sujeto se representa a sí mismo mentalmente. Es, literalmente, lo que le pasa por la cabeza. La proposición en este sentido no es necesariamente aseverada desde un punto de vista lógico, puesto que podemos afirmar proposiciones falsas. 
Lo que realmente nos interesa no son las proposiciones que tienen ser, sino las aseveradas lógicamente, es decir, aquellas que aparte de ser tienen existencia. Las proposiciones aseveradas lógicamente son las proposiciones verdaderas. Podemos entonces sintetizar la posición de Russell como sigue: el estudio de la proposición sólo puede efectuarse a través del análisis lógico de la gramática superficial. Sólo la investigación fundada en la gramática filosófica puede poner al descubierto, por ejemplo, la complejidad y la estructura reales de las proposiciones. Por otra parte, la teoría lógica del significado nos permite inferir que la totalidad de las proposiciones aseveradas y el mundo son una y la misma cosa. Ahora bien, podría argüirse que si nosotros pensamos proposiciones, esto es, que si nuestro pensamiento es básica o fundamentalmente proposicional y las proposiciones asertadas son logicamente el mundo, entonces Russell está en el fondo manteniendo un solipsismo radical: el mundo son mis pensamientos. Afirmar esto, no obstante, sería un error, por la sencilla razón de que, como dije, nosotros también pensamos proposiciones falsas, es decir, algunas de nuestras proposiciones son aseveradas sólo sicológicamente. Por lo tanto, podemos sin temor afirmar que para Russell el pensamiento abarca más que lo existente, o sea, más que el mundo real. Tiene entonces que señalarse también que para él la realidad abarca más que el mundo "real". El pensamiento es de combinaciones de términos y, ocasionalmente, del mundo. Así, pues, la importancia de la filosofía del pensamiento radica entre otras cosas en que nos proporciona verdad metafísica sobre el mundo. Nosotros pensamos proposiciones y, si éstas son verdaderas, lo que pensamos o aprehendemos es el mundo. $O$, para expresar la idea de otra manera, lo que es pensado cuando se piensa con verdad es el mundo. La complejidad de las proposiciones es la complejidad de los elementos que constituyen al mundo. Al igual que para Moore, para Russell no hay intermediarios entre la mente y el mundo. El lenguaje es el vehículo contingente del pensamiento. Dadas las características de los términos (inmutables, indestructibles, etc.), es claro que el pensamiento es independiente de las palabras.

Con la Teoría de las Descripciones, Russell va a tratar de ir todavía más allá en su teoría del pensamiento, pero de hecho va a introducir elementos perturbadores que acabarán finalmente echando por tierra la teoría de la proposición y que pondrán a Russell en el camino del fenomenalismo radical y del construccionismo. Una de las consecuencias de la nueva teoría es el principio de "Acquaintance". Dicho principio fue presentado por Russell de diversas maneras, pero su formulación en español es un tanto difícil de articular. La traducción más adecuada es, quizá, la siguiente: “Toda proposición que podamos comprender debe estar por completo constituida por objetos que conocemos directamente 
en la experiencia (o, también, por objetos que son datos inmediatos de nuestra conciencia)." Es claro, independientemente de la formulación, que mediante el principio, Russell le está dando un giro especial a su teoría abstracta de la proposición y orientándola por la desastrosa senda del empirismo radical. Aquí, aparte de la infinidad de problemas que se le plantean a Russell, deseo señalar que se le puede acusar, en particular, de inconsistencia. Intentaré ahora hacer ver por qué.

El principio de "acquaintance" es un principio reduccionista. Russell nos había dicho, en primer lugar, que nosotros pensamos proposiciones tanto verdaderas (o aseveradas lógicamente) como proposiciones falsas (o aseveradas sólo sicológicamente). Ahora, sin embargo, nos dice que las segundas son reducibles a, es decir, explicables mediante, las primeras. Y va todavía más lejos. Nos dice también que las proposiciones que requieren en su formulación verbal símbolos incompletos son también reducibles a las proposiciones en cuya formulación lingüística aparecen nada más palabras del vocabulario, empírico mínimo. Aquellas proposiciones a las que todas las demás son reducibles son las proposiciones cuyos términos son datos inmediatos de nuestra conciencia o que conocemos directamente en la experiencia, ya sea que los percibamos o que los concibamos. Aquí es donde debe empezar el análisis epistemológico, ya que se tiene que determinar cuáles son las proposiciones que así conocemos. El principio de "acquaintance" es claramente un principio empirista y, a la vez, atomista. Tiene por lo tanto consecuencias decisivas para nuestro tema, porque ya no podremos decir que conocemos entidades complejas, sino sólo sus elementos. La relación cognitiva con dichos elementos es la experiencia en el sentido más estricto, crudo o puro de la palabra. De ahí que lo que Russell está de hecho afirmando es que es la experiencia, así concebida, lo que fija los límites a nuestro pensamiento. No podemos ir en el pensamiento más allá de los límites fijados por nuestra experiencia privada. De esta manera, Russell hace ahora coincidir el análisis del pensamiento con el análisis de la experiencia y, en verdad, con el análisis lógico del mundo. Esto nos da la razón de ser de su programa construccionista. No es posible no señalar que, además de subjetivista a ultranza, la nueva posición es irreconciliable con los supuestos de la teoría original de la proposición, puesto que ahora se hace depender a ésta, lógica, ontológica y epistemológicamente, de sus elementos.

Por razones conectadas con la noción de verdad, Russell se sintió obligado a abandonar la creencia en la existencia de las proposiciones, consideradas no sólo como entidades objetivas sino también como objetos de pensamiento. Una de sus motivaciones, sin embargo, es claramente infructuosa. Russell justifica su abandono de la creencia en la existencia 
de las proposiciones arguyendo que no puede haber en el mundo proposiciones falsas y dado que no hay diferencia esencial entre las proposiciones verdaderas y las falsas ( $q u a$ proposiciones), concluye que no puede haber proposiciones en absoluto. Modifica entonces su ontología y opta de esta manera por una ontologia de hechos, pero casi de inmediato se ve obligado a incluir hechos negativos y es en verdad difícil, por no decir imposible, comprender por qué estos últimos serían menos paradójicos o menos extraordinarios o más inteligibles que las proposiciones falsas. Por otra parte, vale la pena notar que, a pesar de toda la batería de argumentos desplegada en contra de las proposiciones, de hecho Russell siguió aceptándolas como algo real. Esto es lo que ahora trataré de hacer ver.

El papel que el manuscrito "Teorfa del Conocimiento" tenía que representar en la obra de Russell era el de un punto de partida completamente nuevo en la investigación epistemológica. En dicho manuscrito Russell ya no se pregunta '¿qué son las proposiciones?', sino que se aboca más bien a dar respuesta a la pregunta '¿Qué significa "comprender una proposición"?' Desde esta nueva perspectiva, la elucidación total de la proposición habría de resultar de la conjunción de por lo menos las siguientes teorías:

a) Una teoría de semántica o de lógica filosófica que revelara las condiciones epistémicas de la significatividad.

b) Una teoría del conocimiento que diera cuenta de casos concretos de comprensión, es decir, de lo que anteriormente habría podido ser llamado 'actitudes proposicionales' (creer, dudar, afirmar, negar, etc.). Aquí nos vamos a ocupar únicamente de la primera parte de la doctrina que es, por así decirlo, su esqueletó lógico.

El primer punto en el que Russell insiste es que todas las actitudes proposicionales presuponen a la comprensión y que, y éste es su segundo punto, la comprensión presupone o se funda en el conocimiento directo (acquaintance). Supongamos que estamos frente a un complejo $a R b$. De acuerdo con Russell, $a, b$ y $R$ tienen que ser datos de la conciencia. Aquí surge ya un problema porque $R$, siendo una relación, tiene un sentido y puede por lo tanto dar lugar a dos complejos distintos. Si nos dan la lista de los elementos, no podríamos determinar a priori si el complejo que analizamos es $a R b$ o $b R a$. Para evitar esta dificultad, Russell sugiere que consideremos el caso de una relación simétrica. Aquí, obviamente, el problema ya no se plantea dado que la relación $x R y$ es simétrica si y sólo si $x R y \leftrightarrow y R x$. Ahora bien, Russell insiste en que la mera relación cognitiva con los elementos del complejo (sensación y con- 
cepción puras) no basta. Después de todo, la lógica también es necesaria para que se produzca el fenómeno de comprensión. Aquí Russell se adelanta al Tractatus: no podemos pensar ilógicamente. Russell interpreta esto de la siguiente manera: tenemos que conocer directamente la forma lógica de la relación dual. Si no conociéramos dicha forma no podríamos conocer o saber que una relación determinada, es decir, un universal, está de hecho relacionando a dos términos. Dicha situación sería ininteligible e imposible para nosotros. En nuestro esfuerzo por conocer el mundo no podemos nosotros ignorar su forma lógica. Ésta es representada simbólicamente no mediante una palabra o una expresión ad hoc, sino que es aquello de lo que hablamos cuando usamos única y exclusivamente variables, puesto que en lógica no se habla de nada particular. La forma lógica es lo que tienen en común todos los complejos (en este caso, duales). Así, llegamos a ella de la siguiente manera:
a) $a R b$
b) $x R b$
c) $x R y$
d) $\quad x \emptyset y$
e) (马x) (घy) $x \emptyset y$

Traducido al lenguaje natural, '(e)' significa: 'La forma lógica de la relación dual es: "algo relacionado con algo, algo mantiene una relación determinada con algo".' Russell nos ofrece, asimismo, la siguiente representación gráfica:

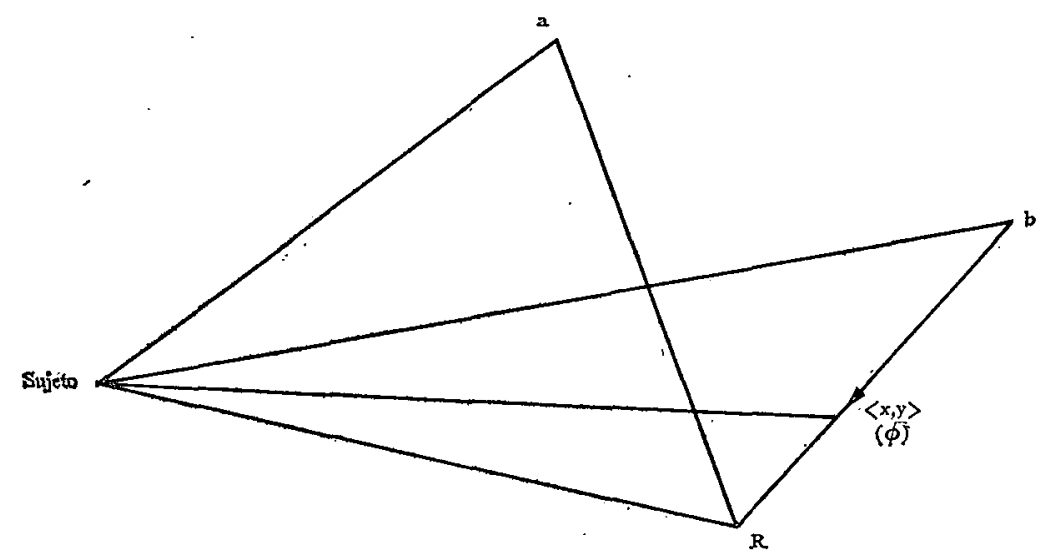

Una vez hechas estas aclaraciones, Russell avanza su nueva definición de 'proposición'. Ésta es: hay un sujeto y un acto o una instancia del fenómeno de comprensión tales que 


$$
\begin{aligned}
& C(S, a, b, R,<x, y>) \text { ó } \\
& C(S, a, b, R, \emptyset)
\end{aligned}
$$

Es evidente que esta nueva concepción no es una concepción acabada, bien redondeada, si bien si ilumina algunos aspectos del conocimiento empírico. Está, no obstante, claramente expuesta a ciertas objeciones. Russell mismo señala una de ellas y abiertamente reconoce que no ve solución o manera de eludirla. La objeción consiste en que al hacer de la proposición una función de la comprensión, es decir, de algo mental, resulta que la definición no cubre más que a las proposiciones de hecho pensadas o mencionadas por nosotros. Esto, sin embargo, equivale de hecho a la adopción o a la postulación del solipsismo. Es esto lo que está a la base de las ulteriores "construcciones lógicas" de Russell: éstas son el resultado de su esfuerzo por evadir precisamente dicho solipsismo, al que se ve comprometido. Un segundo problema es que, de una u otra manera, Russell vuelve a reconocer a la proposición como algo real y la prueba de ello es que una cuantificación está involucrada.

A pesar de que pueden elevarse ésta y otras objeciones en contra de la nueva doctrina, no creo que todo en ella sea rechazable ni que todo en ella sea incompatible con lo que Russell habia dicho anteriormente. Creo que con ella, Russell hace una aportación fundamental: se introduce y se hace valer a la noción de forma lógica. Hasta Los Problemas de la Filosofia, Russell se había limitado a hablar de particulares, propiedades y relaciones. Su concepción de la proposición se ve, pues, enriquecida con la adición de las formas lógicas. Pero, obviamente, el descubrimiento de las formas lógicas no podría ser usado para rechazar la idea misma de proposición. Más bien todo lo contrario.

Nuestra tarea será ahora tratar de construir una teoría completa y coherente del pensamiento con los elementos que Russell nos da y, segundo, someter dicha teoría a crítica.

Para empezar, creo que será instructivo enunciar por lo menos algunas de las diferencias entre las diversas teorías que hemos mencionado. Frente a la de Moore, la teoría de Russell es superior por razones más bien obvias. En primer lugar, Moore no reconoce distinciones de tipo: todos los conceptos están, por así decirlo, al mismo nivel. Pero esto hace difícil comprender cómo su conjunción puede dar lugar a una proposición o a un juicio. Parece inevitable admitir que, en algún sentido, los predicados tienen que estar "insaturados", a diferencia de lo que pasa con las "cosas". En este punto, Frege y Russell coinciden. La teoría de Moore está inevitablemente expuesta a un argumento del tipo "Tercer Hombre", puesto que no distingue entre relaciones y objetos. Por otra parte, Moore, Russell y Frege coinciden en ver a los conceptos y a los 
términos nombrados como entidades inmutables, eternas, etc. En lo que difieren es en sus respectivas caracterizaciones de la relación que se da entre nuestro pensamiento y sus objetos. Para Moore, como quedó dicho, pensamiento y mundo son una y la misma cosa, si bien no hay que identificar pensamiento y secuencia de ideas. Para Frege, el mundo de los conceptos o sentidos forma un tercer mundo, distinto del mundo sensorial si bien de alguna manera relacionado con él. En relación con esta cuestión, Russell y Frege marchan de nuevo al unísono. Russell divide a la realidad en el mundo de la materia, el mundo de lo mental y el mundo de los universales y este último es el tercer mundo de Frege. Sus proposiciones, como las de Frege, están estructuradas. Vale la pena notar, sin embargo, que no hay nada en la doctrina de Moore, dejando de lado la cuestión de la jerarquía de tipos, que le impida integrarse a las de Russell y Frege. Estoy hablando, claro está, de un Russell sumamente raro y desde luego de un Russell no "oficial". Esto requiere ciertas aclaraciones.

El gran problema de Russell es provocado por su adhesión al empirismo y al análisis. La teoría formal de las proposiciones, enriquecida por su trilogia "particular-universal-forma lógica", es perfectamente coherente y en principio no hay problema con ella. Pero con la interpretación que Russell hace de-su Teoría de las Descripciones, se introduce un elemento discordante. El problema es, como vimos, que, según Russell, una de las consecuencias de dicha teoría es el principio de acquaintance y un problema que dicho principio entraña es, como ya dije, que se trata indudablemente de un principio reduccionista, es decir, tiene consecuencias ontológicas. Así, si lo que el principio afirma es que unas proposiciones son reducibles a otras, ello debe querer decir que, estrictamente hablando, las primeras no existen, no son nada real. Se trata, claro está, de todas aquellas proposiciones para cuya formulación verbal hubo que emplear símbolos incompletos. Queda entonces claro que, debido al uso del principio mencionado, la teoría de la proposición tiene un alcance muchísimo más limitado que lo que habría podido suponerse en un principio: vale, si vale, única y exclusivamente para los casos de proposición cuyos constituyentes conocemos directamente. Dicho de otro modo, vale únicamente para las proposiciones atómicas ( $\mathrm{y}$ tal vez para ciertas proposiciones generales). Se le plantean, pues, a Russell los siguientes problemas: tiene que dar cuenta de nuestro conocimiento molecular (el negativo incluido) y del conocimiento "por descripción". Eso hasta cierto punto lo logra, por lo menos formalmente, pero: 1) introduce hechos negativos y 2) se ve comprometido con un fenomenalismo radical y por consiguiente con un programa construccionista del cual no hay salida, ni fácil ni difícil. 
El origen de este problema es el deseo de Russell de conciliar una metafísica de corte idealista (la doctrina de las proposiciones) con el empirismo. Creo que, hasta cierto punto, Russell obtiene lo que quiere, pero el precio es sumamente elevado, porque si Russell hace valer su principio del conocimiento directo, entonces las posibilidades de nuestro pensamiento se ven sumamente restringidas y parecemos perder una gran cantidad de cosas que normalmente estamos inclinados o dispuestos a llamar 'pensamiento genuino', independientemente de cualquier reducción. Y no podemos re-interpretar el principio de manera menos drástica o más blanda, dando cabida aś a lo que mediante él se explica como redundante, porque entonces dicho principio pierde todo atractivo y se vuelve una trivialidad. Nótese también que de acuerdo con la caracterización russelliana de lo empírico, la sensación se convierte en la esencia del pensar o, por lo menos, del pensar sintético y esta conclusión es altamente dudosa y cuestionable. Está también el problema del solipsismo: si las proposiciones que yo pienso constituyen el mundo y ellas mismas están constituidas únicamente por entidades que yo conozco directamente, entre otros mis datos sensoriales, entonces es lógicamente imposible que alguien más piense las mismas proposiciones que yo. De igual manera, resulta ahora imposible que yo me represente algo que rebase los límites del conocimiento básico o sensorial. Para mí, en este caso, no hay mundo externo.

¿Cómo evaluar las teorías de Russell? ¿Se puede acaso elaborar con el material que él nos proporciona una teoría coherente y completa del pensamiento? Sintetizando lo que hasta aquí hemos dicho y basándonos en lo que Russell nos dice, creo que podemos construir la siguiente "picture" del pensamiento: nosotros pensamos proposiciones que son los constituyentes del mundo, es decir, el mundo es la totalidad de las proposiciones, no de los términos. Las proposiciones se componen de una variedad de "elementos" como lo son particulares, universales y formas lógicas. Las proposiciones son objetivas. Ahora bien, el análisis del fenómeno cognitivo del pensar, el pensamiento cartesiano, revela que cada quien piensa únicamente lo que conoce directamente y es claro que cuando están involucrados particulares nadie puede pensar las mismas proposiciones que otros. Por lo tanto, lo único que pienso, por lo menos cuando pienso acerca del "mundo empírico", son los contenidos de mi actividad, los cuales no son independientes de mí. Pienso, por lo tanto, los productos de mi mente. El mundo externo es entonces mi construcción. Si éste fuera en verdad el resultado al que lleva la doctrina, habría que concluir que Russell no tiene una teoría completa sobre el pensamiento y que en su doctrina una de sus partes necesariamente se desarrolla en detrimento de la otra. 